\title{
Effective mass of electron in monolayer graphene: Electron-phonon interaction
}

E. Tiras, S. Ardali, T. Tiras, E. Arslan, S. Cakmakyapan, O. Kazar, J. Hassan, E. Janzén, and E. Ozbay

Citation: Journal of Applied Physics 113, 043708 (2013);

View online: https://doi.org/10.1063/1.4789385

View Table of Contents: http://aip.scitation.org/toc/jap/113/4

Published by the American Institute of Physics

\section{Articles you may be interested in}

Inverse relationship between carrier mobility and bandgap in graphene

The Journal of Chemical Physics 138, 084701 (2013); 10.1063/1.4792142

Diffusion coefficient, correlation function, and power spectral density of velocity fluctuations in monolayer graphene

Journal of Applied Physics 114, 143702 (2013); 10.1063/1.4824182

Mobility and saturation velocity in graphene on $\mathrm{SiO}_{2}$

Applied Physics Letters 97, 082112 (2010); 10.1063/1.3483130

Temperature dependent carrier mobility in graphene: Effect of Pd nanoparticle functionalization and hydrogenation

Applied Physics Letters 108, 093102 (2016); 10.1063/1.4942975

Electron mobility calculation for graphene on substrates

Journal of Applied Physics 116, 083703 (2014); 10.1063/1.4893650

First-principles calculation of intrinsic carrier mobility of silicene

Journal of Applied Physics 114, 093712 (2013); 10.1063/1.4820526

\section{Scilight}

Sharp, quick summaries illuminating the latest physics research

\section{Sign up for FREE!}

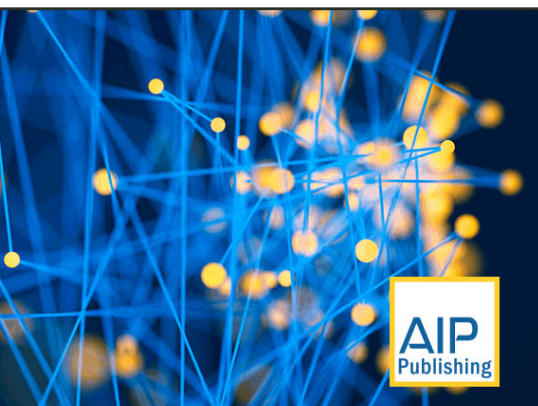




\title{
Effective mass of electron in monolayer graphene: Electron-phonon interaction
}

\author{
E. Tiras, ${ }^{1, a)}$ S. Ardali, ${ }^{1}$ T. Tiras, ${ }^{1}$ E. Arslan, ${ }^{2}$ S. Cakmakyapan, ${ }^{2}$ O. Kazar, ${ }^{2}$ J. Hassan, ${ }^{3}$ \\ E. Janzén, ${ }^{3}$ and E. Ozbay ${ }^{2}$ \\ ${ }^{1}$ Department of Physics, Faculty of Science, Anadolu University, Yunus Emre Campus, \\ 26470 Eskisehir, Turkey \\ ${ }^{2}$ Nanotechnology Research Center, Departments of Physics and Electrical and Electronics Engineering, \\ Bilkent University, Bilkent, 06800 Ankara, Turkey \\ ${ }^{3}$ Departments of Physics, Chemistry and Biology, Linkoping University of Technology, S-581 83 Linkoping, \\ Sweden
}

(Received 4 September 2012; accepted 8 January 2013; published online 25 January 2013)

\begin{abstract}
Shubnikov-de Haas $(\mathrm{SdH})$ and Hall effect measurements performed in a temperature range between 1.8 and $275 \mathrm{~K}$, at an electric field up to $35 \mathrm{kV} \mathrm{m}^{-1}$ and magnetic fields up to $11 \mathrm{~T}$, have been used to investigate the electronic transport properties of monolayer graphene on $\mathrm{SiC}$ substrate. The number of layers was determined by the use of the Raman spectroscopy. The carrier density and in-plane effective mass of electrons have been obtained from the periods and temperature dependencies of the amplitude of the $\mathrm{SdH}$ oscillations, respectively. The effective mass is in good agreement with the current results in the literature. The two-dimensional (2D) electron energy relaxations in monolayer graphene were also investigated experimentally. The electron temperature $\left(T_{e}\right)$ of hot electrons was obtained from the lattice temperature $\left(T_{L}\right)$ and the applied electric field dependencies of the amplitude of $\mathrm{SdH}$ oscillations. The experimental results for the electron temperature dependence of power loss indicate that the energy relaxation of electrons is due to acoustic phonon emission via mixed unscreened piezoelectric interaction and deformation-potential scattering. (C) 2013 American Institute of Physics. [http://dx.doi.org/10.1063/1.4789385]
\end{abstract}

\section{INTRODUCTION}

The discovery of graphene, a dense honeycomb twodimensional structure formed by a monolayer or a few layers of carbon atoms, has led to the intense research activities aimed at understanding the electronic properties of this truly two-dimensional (2D) electronic system. ${ }^{1}$ It is a massless and gapless Dirac quasi-particle system with roughly linear energy dispersion. Its unique physical properties make it an ideal system to look for new physics, and it is a promising candidate for applications from gas sensors to touch screens.

The Shubnikov-de Haas ( $\mathrm{SdH}$ ) oscillations in magnetoresistance have been extensively in the investigations of the electronic transport properties of 2D structures at low temperature. The SdH effect has been proven to be a powerful tool for determining the in-plane effective mass $\left(m^{*}\right)$ and electron-phonon interactions. ${ }^{2-5}$ Recently, the electron effective mass in monolayer graphene has been obtained from the temperature dependence of the amplitude of $\mathrm{SdH}$ oscillations. ${ }^{6-8}$ They reported the electron effective mass of graphene samples depending on the square root of the $2 \mathrm{D}$ carrier concentration.

The determination of the temperature of electrons, under electric-field heating conditions in the steady state, provides useful information about the electron-phonon interactions involved in the energy relaxation process. Electron energy loss rates in graphene samples have been investigated

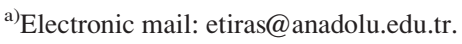

theoretically $^{9-12}$ and experimentally. ${ }^{8,13-18}$ Most previous experimental works on energy loss rates in graphene have been conducted using optical excitation at much higher energies $^{13-15}$ with non-equilibrium carrier distributions and carrier temperatures in excess of $5000 \mathrm{~K}$ where the carrier lifetime has saturated. In addition, the electron energy loss rates in graphene samples have been investigated using electrical measurements performed in a high-current regime at temperatures between 4 and $250 \mathrm{~K} .{ }^{16,17}$ Recently, Tan et al. ${ }^{8}$ and Baker et al. ${ }^{18}$ investigated the carrier temperature dependence of the energy loss rate in graphene by using the amplitude of Shubnikov de Haas oscillations as a function of the electric field. It was reported that the carrier energy loss in graphene found to be proportional to fourth order of the electron temperature at carrier temperatures up to $100 \mathrm{~K}$, indicating that the energy relaxation of electrons is due to acoustic phonon emission via deformation potential coupling. ${ }^{18,19}$

An in-depth understanding of the fundamental optical and electronic properties is yet to be established for the design and development of functional devices. The objective of this work is to determine the in-plane effective mass of electrons in monolayer graphene. This is achieved by systematically measuring the temperature dependence of Shubnikov de Haas $(\mathrm{SdH})$ oscillations in magnetoresistance for monolayer graphene. Furthermore, the temperature of the hot electrons $\left(T_{e}\right)$ of the monolayer graphene sample and the corresponding power loss $(P)$ have been determined as a function of the applied electric field using the $\mathrm{SdH}$ effect method. 


\section{EXPERIMENTAL PROCEDURE}

Experiments were carried out on the epitaxial graphene grown on $(10 \times 10) \mathrm{mm}^{2}$ nominally on-axis $4 \mathrm{H}-\mathrm{SiC}(0001)$, Si-face chemo-mechanically polished substrates. All the substrates were obtained from a single 4-in. wafer to avoid the influence of large variations of unintentional off-cut from wafer to wafer. Three samples were grown during each growth run to have similar graphene. This was mainly to facilitate device processing and to understand the influence of different processing steps on the electrical properties of graphene. The optimized surface preparation and growth process were used to obtain 1-2 layers of graphene. In situ etching, graphene growth, and hydrogen intercalation were performed on samples during the same and single growth sequence without exposing samples to air. A unique in situ surface preparation method was adopted from the on-axis homoepitaxial growth of $4 \mathrm{H}-\mathrm{SiC}$. The samples were exposed to a mixture of silane and hydrogen $(0.006 \%$ silane in hydrogen) for $10 \mathrm{~min}$ at $1400{ }^{\circ} \mathrm{C}$. The following graphene growth was performed in vacuum $\left(5-9 \times 10^{-6} \mathrm{mbar}\right)$ at $1400^{\circ} \mathrm{C}$ for $1 \mathrm{~h}$. After graphene growth, the samples were cooled to below $500^{\circ} \mathrm{C}$ in vacuum. The growth cell was then filled with hydrogen to a pressure of $500 \mathrm{mbar}$, and the intercalation process was made at $700^{\circ} \mathrm{C}$ for $1 \mathrm{~h}$. For the transport measurements by using the Hall bar geometry, we have designed and fabricated a photomask with electron beam lithography in order to perform each fabrication step with optical lithography. Ohmic contacts were fabricated with the reverse lithography technique. After the development, $20 \mathrm{~nm}$ titanium and $100 \mathrm{~nm}$ gold were deposited by an electron beam evaporator, followed by the standard liftoff process. The mesa lithography step was performed in order to preserve the active graphene region, while etching the rest of the graphene on the surface with $\mathrm{O}_{2}$ plasma. After the etching process, the $500 \mu \mathrm{m}$ by $1100 \mu \mathrm{m}$ active graphene region was obtained. Interconnect metal lithography was performed by using a $30 \mathrm{~nm} / 220 \mathrm{~nm} \mathrm{Ti} / \mathrm{Au}$ metal pair. Finally, devices were bonded for Hall measurements.

The longitudinal resistance $\left(R_{x x}\right)$ along the applied current measurements were carried out as functions of (i) the applied electric field $F$ at a fixed lattice temperature $T_{L 0}$ and (ii) the lattice temperature $T_{L}$ at a fixed electric field $F_{0}$ that was low enough to ensure ohmic conditions and hence to avoid carrier heating. In the experiments, a conventional dc technique in combination with a constant current source (Keithley 2400) and a nanovoltmeter (Keithley 2182 A) in a cryogen free superconducting magnet system (Cryogenics Ltd., Model No. J2414) were used. The current (I) flow was in the plane of the electron gas. Steady magnetic fields up to $11 \mathrm{~T}$ were applied perpendicular to the plane of the samples and, therefore, to the plane of 2D electron gas. All the measurements were taken in the dark. The $R_{x x}$ and the Hall resistance $\left(R_{x y}\right)$ were measured as a function of temperature from 1.8 to $275 \mathrm{~K}$. A static magnetic field $(B=1 \mathrm{~T})$ was applied to the sample perpendicular to the current plane. The Hall mobility $\left(\mu_{H}\right)$ and the sheet carrier density $\left(N_{H}\right)$ were obtained using the following equations:

$$
R_{x y}=\frac{B}{N_{H} e},
$$

$$
\mu_{H}=\frac{L}{b N_{H} e R_{x x}}=\frac{e \tau_{t}}{m^{*}},
$$

where $\tau_{t}$ is the transport lifetime, $b(=0.6 \mathrm{~mm})$ and $L$ $(=1 \mathrm{~mm})$ are the width and length of the Hall bar, respectively. The applied electric field was also obtained using the longitudinal resistance measured at $B=0 \mathrm{~T}\left(R_{x x}(B=0)\right.$ $=6113 \Omega)$ in the following equation:

$$
F=\frac{R_{x x}(B=0)}{L} .
$$

In the applied electric field dependent magnetoresistance measurements, applied current was applied along the length of the sample in the range of $I=1 \mu \mathrm{A}$ to $8 \mathrm{~mA}$.

The Raman spectra were obtained at room temperature by using the Bruker Optics FT-Raman Scope III system. As an excitation source, a wavelength of $633 \mathrm{~nm}(1.96 \mathrm{eV})$ was applied in the sample growth direction.

\section{RESULTS AND DISCUSSION}

Figure 1 shows the room-temperature Raman spectra for the graphene recorded in the grown-axis backscattering configurations. For the graphene layer on $\mathrm{SiC}$, it is apparently difficult to distinguish the $\mathrm{G}$ and $\mathrm{D}$ peaks in the spectra because their intensities are much smaller than those of the surrounding peaks from the $\mathrm{SiC}$ substrate. ${ }^{20}$ The sharp and strong peak at $775.0 \mathrm{~cm}^{-1}$ is the $\mathrm{E}_{2}$ planar optical, $962.5 \mathrm{~cm}^{-1}$ is an $\mathrm{A}_{1}$ longitudinal optical (LO) mode, and $201.5 \mathrm{~cm}^{-1}$ which is an $\mathrm{E}_{2}$ planar or transverse acoustic mode of SiC. ${ }^{21}$ The defectinduced D band peak, which should be near $1350 \mathrm{~cm}^{-1}$, is absent for these layers, attesting to the high crystalline quality of graphene layers. ${ }^{20}$ The small $\mathrm{G}$ peak is also located in between the $\mathrm{SiC}$ peaks at 1500 and $1700 \mathrm{~cm}^{-1} \cdot{ }^{20}$ Only the $2 \mathrm{D}$ peaks are well isolated around $2600-2750 \mathrm{~cm}^{-1}$ and are out of the range of the $\mathrm{SiC}$ peaks. ${ }^{22}$ It has been shown that the Raman scattering fingerprint of graphene mono and multi layers lies in the 2D feature, whose shape strongly depends on the interlayer coupling. Graphene shows a single component $2 \mathrm{D}$ band while a multi-components $2 \mathrm{D}$ band is observed upon increasing the number of layers in graphitic structures. In our sample, the 2D peak at $2668.5 \mathrm{~cm}^{-1}$ indicates that the sample is a monolayer of graphene.

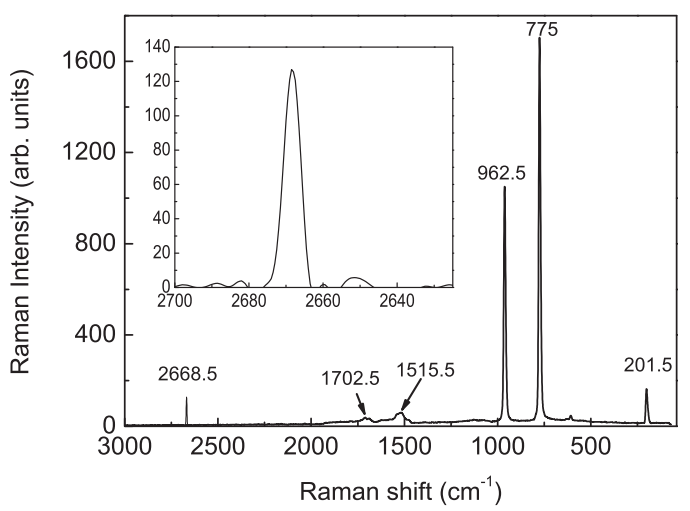

FIG. 1. Raman spectra in monolayer graphene on SiC substrate. The enlarged 2D-band region is also shown in the inserted figure. 
The temperature dependence of the sheet carrier density and Hall mobility in the graphene is plotted in Fig. 2. At low temperatures, the sheet carrier density remains practically constant up to a temperature of $100 \mathrm{~K}$. At higher temperatures, the sheet carrier density increases monotonically with increasing temperature possibly due to thermally generated carriers located outside the channel. A similar behavior of carrier density with temperature, although less pronounced, was reported previously for 2D structures such as modulation-doped $\mathrm{GaAs} / \mathrm{Ga}_{1-x} \mathrm{Al}_{x} \mathrm{As}$ heterojunctions. ${ }^{23-25}$ The Hall mobility of electrons in the graphene sample increases monotonically with a decreasing temperature from room temperature, begins to level off at about $100 \mathrm{~K}$, and saturates at about $50 \mathrm{~K}$ (see Fig. 1). This behavior reflects the $2 \mathrm{D}$ character of the electrons in the channel. ${ }^{26}$

Figure 3 shows a typical example of the magnetoresistance $R_{x x}(B)$ and Hall resistance $\left(R_{x y}\right)$ measured at $1.8 \mathrm{~K}$. $\mathrm{SdH}$ oscillations and Hall plateau are clearly visible over the magnetic field range between $B=2$ and $7 \mathrm{~T}$ and no higher harmonics are apparent in the $\mathrm{SdH}$ oscillations. The overall positive $R_{x y}(B)$ indicates that the contribution is mainly from electrons. At a high magnetic field, $R_{x y}(B)$ exhibits plateau and $R_{x x}(B)$ is vanishing, which is the hallmark of the quantum hall effect $(\mathrm{QHE}){ }^{7}$ At least two well-defined plateaus with values $\left(h / 2 e^{2}=12893 \Omega\right)$ and $\left(h / 6 e^{2}=4300 \Omega\right)$, followed by a developing $\left(h / 10 e^{2}=2581 \Omega\right)$ plateau, are observed before the QHE features transform into $\mathrm{SdH}$ oscillations at a lower magnetic field. The plateaux numbers observed follow the $2+4 v(v=0,1,2$,. is the filling factor of the quantum Hall states) pattern which confirms that our sample is monolayer graphene. ${ }^{18,27,28}$

In order to check the 2D nature of the electron gas giving rise to the quantum oscillations in magnetoresistance, measurements were also performed as a function of the angle $\theta$ between the normal to the plane of the $2 \mathrm{D}$ electron gas and the applied magnetic field. It was found that the peak position shift with a factor of $\cos \theta$ and the oscillations disappear at $\theta=80^{\circ}$ (Fig. 4). This observation is a characteristic of 2D electron gas. ${ }^{29}$

Figures 5(a) and 5(b) show typical examples for the magnetoresistance $R_{x x}(B)$ measurements in the graphene sample for different lattice temperatures at a fixed electric

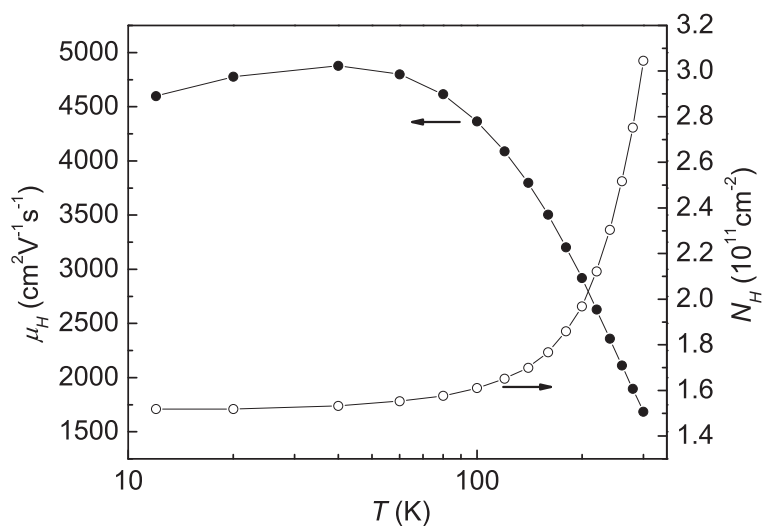

FIG. 2. Temperature dependence of the sheet carrier density $\left(N_{H}\right)$ and Hall mobility $\left(\mu_{H}\right)$ of electrons in the graphene.

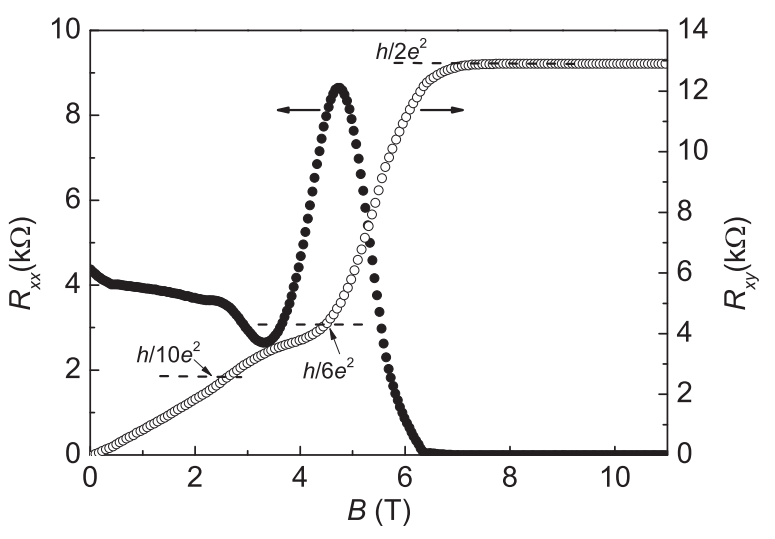

FIG. 3. The magnetoresistance $\left(R_{x x}(B)\right)$ and Hall resistance $\left(R_{x y}(B)\right)$ for graphene sample measured at $1.8 \mathrm{~K}$. The horizontal lines correspond to $h / v e^{2}$ values. The QHE in the electron gas is demonstrated by only one quantized plateau in $R_{x y}$ with vanishing $R_{x x}$ in the corresponding magnetic field regime.

$F=4.43 \mathrm{~V} \mathrm{~m}^{-1}$, and field and for different electric fields at a fixed lattice temperature $T_{L}=1.8 \mathrm{~K}$, respectively. The data clearly show the decrease in the amplitude of the SdH oscillations with an increasing lattice temperature or electric field. The peak positions of the oscillations are also shifted to a higher magnetic field with an increasing temperature or electric field. Similar behaviors have also been observed for monolayer graphene in the literature. ${ }^{7,18}$ The magnetic field of the initial value of the Hall plateau and the zero value of $R_{x x}(B)$ at a high magnetic field shift to a higher magnetic field with an increasing temperature and electric field.

The period of the $\mathrm{SdH}$ oscillations has been obtained from the Fourier analysis. The Fourier analysis of the $\mathrm{SdH}$ oscillations (Fig. 6) confirms that only the first subband is populated and that the contribution of higher harmonics is insignificant. The $2 \mathrm{D}$ carrier density $\left(N_{2 D}\right)$ of the graphene sample can be calculated using the oscillation period ${ }^{30,31}$

$$
\Delta\left(\frac{1}{B_{n}}\right)=\frac{e}{\pi \hbar N_{2 D}},
$$

where $e$ and $\hbar(=h / 2 \pi)$ are the electron charge and Planck's constant, respectively. The results for the graphene sample is

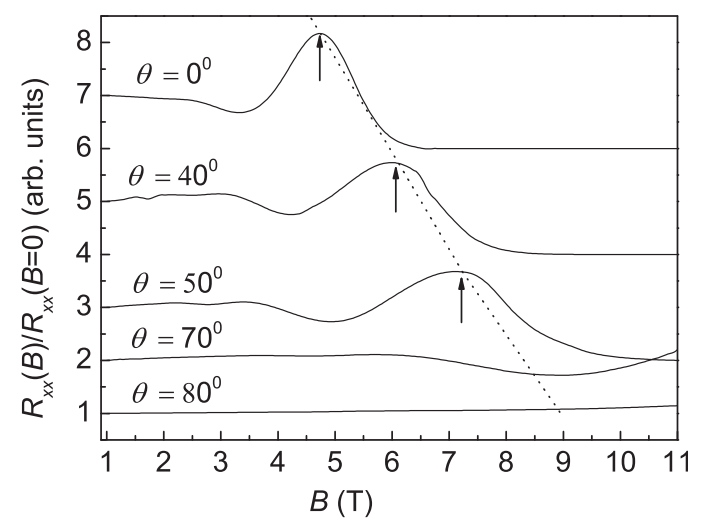

FIG. 4. The normalized magnetoresistance $R_{x x}(B) / R_{x x}(B=0)$ measured at $1.8 \mathrm{~K}$ as a function of the angle $\theta$ between the normal to the plane of the $2 \mathrm{D}$ electron gas and the applied magnetic field. The vertical arrows and dotted lines indicate the angle dependence of the peak position and shifting of the $\theta=0$ peak position of the $\cos \theta$ function, respectively. 

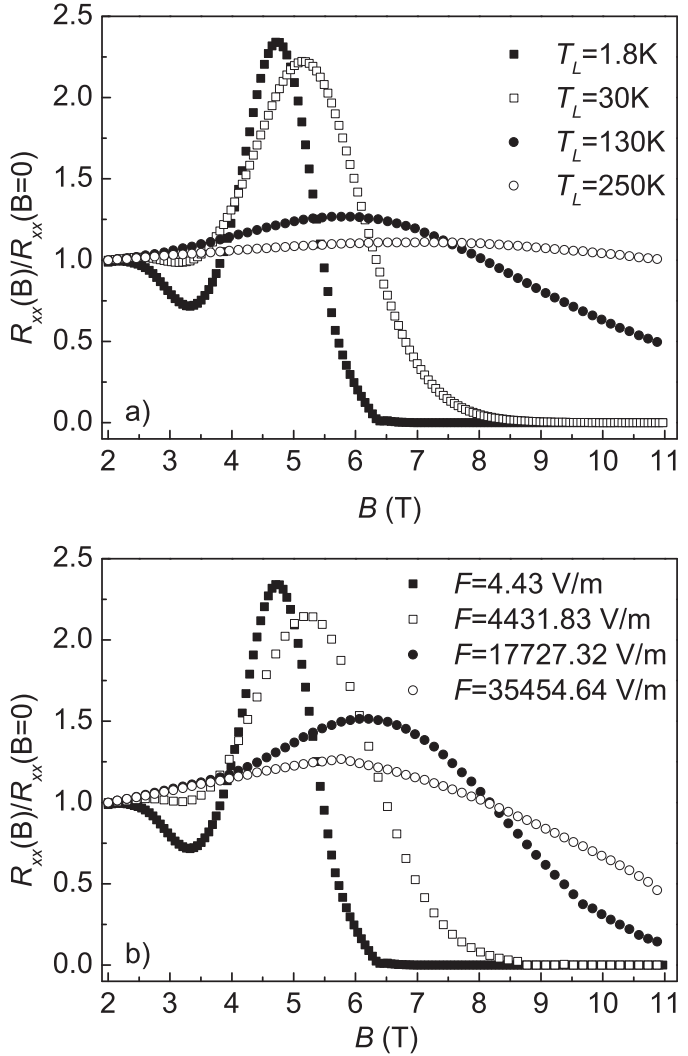

FIG. 5. The effects of temperature (a) and applied electric fields (b) on the $\mathrm{SdH}$ oscillations for the graphene sample.

$N_{2 D}=1.51 \times 10^{15} \mathrm{~m}^{-2}$. The $2 \mathrm{D}$ carrier density is found to be essentially independent of the lattice temperature in the range from $T_{L}=1.8$ to $250 \mathrm{~K}$. In our sample, the ratio $N_{H} / N_{2 D}$ is about one within the experimental accuracy. This result indicates that only $2 \mathrm{D}$ electron densities contribute to the transport properties and, therefore, parallel conduction is negligible. The effective mass $m^{*}$ can be extracted from the temperature dependence of the $\mathrm{SdH}$ amplitude at a constant magnetic field using ${ }^{30,31}$

$$
\frac{A\left(T, B_{n}\right)}{A\left(T_{0}, B_{n}\right)}=\frac{T \sinh \left(2 \pi^{2} k_{B} T_{0} m^{*} / \hbar e B_{n}\right)}{T_{0} \sinh \left(2 \pi^{2} k_{B} T m^{*} / \hbar e B_{n}\right)},
$$

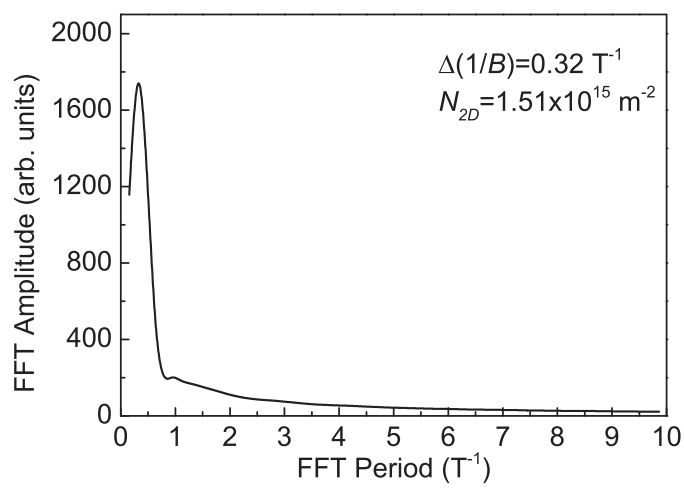

FIG. 6. The fast Fourier spectrum of the oscillations. There is no evidence for the population of higher subbands or for any contribution from higher harmonics. where $A\left(T, B_{n}\right)$ and $A\left(T_{0}, B_{n}\right)$ are the amplitudes of the oscillation peaks observed at a magnetic field $B_{n}$ and at temperatures $T$ and $T_{0}$. The relative amplitudes, $A\left(T, B_{n}\right) / A\left(T_{0}, B_{n}\right)$, are plotted in Fig. 7(a). The relative amplitude decreases with an increasing temperature as a result of thermal damping. ${ }^{5,32}$ The effective mass of $2 \mathrm{D}$ electrons is then determined by fitting the experimental data for the temperature dependence of $A\left(T, B_{n}\right) / A\left(T_{0}, B_{n}\right)$ to Eq. (5). We find $m^{*}=0.012 m_{0}\left(m_{0}\right.$ is the free electron mass). We obtained similar values for all the magnetic fields of measurements, thereby strongly suggesting that the effective mass is essentially independent of the magnetic field. Our electron effective mass for the monolayer graphene sample agrees with the calculated effective mass $m^{*}=\left(\hbar / v_{F}\right) \sqrt{\pi N_{2 D}}$, where $N_{2 D}$ is obtained from the measured $\mathrm{SdH}$ period and $v_{F}=1.1 \times 10^{6} \mathrm{~ms}^{-1}$ is the band velocity adopted from the literature. ${ }^{7,8}$

The SdH oscillations and classical low-field Hall-effect measurements allow for the determination of both the quantum and transport lifetimes of the electrons in the graphene and hence to investigate the relative importance of various scattering mechanisms. The quantum lifetime can be determined from the magnetic-field dependence of the amplitude of the SdH oscillations (i.e., Dingle plots) at a constant temperature provided that the electron effective mass is known $^{26,30,31}$

$$
\ln \left[\frac{A\left(T, B_{n}\right) \cdot B_{n}^{-1 / 2} \cdot \sinh (\chi)}{\chi}\right]=C-\frac{\pi m^{*}}{e \tau_{q}} \frac{1}{B_{n}},
$$
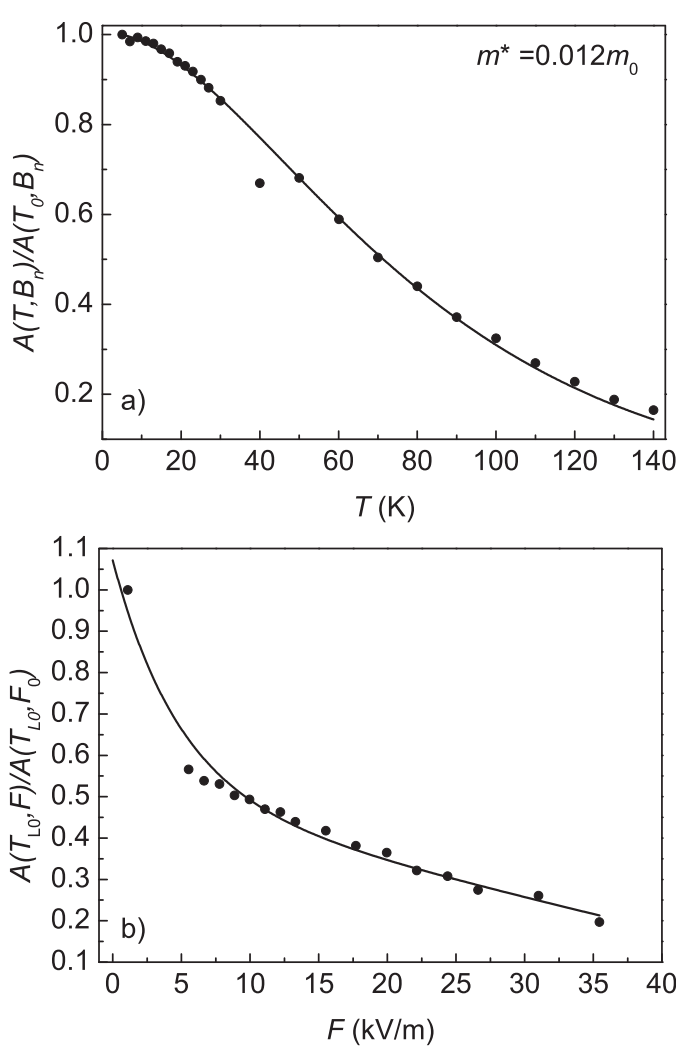

FIG. 7. (a) Temperature and (b) electric-field dependencies of the normalized amplitude of the oscillation peak at $B_{n}$ measured in the graphene sample. The data points represented by the full circles correspond to the $\mathrm{SdH}$ oscillations. The full curve in (a) is the best fit of Eq. (5) to the experimental data. The full curve in (b) is intended as a guide. 
where $C$ is a constant. Figure 8 shows typical examples of the Dingle plots for the samples investigated. There is good agreement between the experimental data and the straight line described by Eq. (6). The quantum lifetime obtained from the slope of the Dingle plot is $\tau_{q}=23$ fs. These values remain constant within $2 \%$ in the whole temperature and magnetic-field ranges of the measurements. The ratio of the quantum to transport lifetime, $\tau_{t} / \tau_{q}$, in our samples is bigger than unity $\left(\tau_{t} / \tau_{q}=1.36\right)$. Theoretical calculations relating the 2D single-particle scattering time (quantum lifetime) to the momentum relaxation time (transport lifetime) predict the $\tau_{t} / \tau_{q}$ ratio of equal to or less than unity for wide-angle scattering and greater than unity for small-angle scattering in the extreme quantum limit for single subband occupancy. ${ }^{26}$ This implies that in our sample the electron scattering with small-angle scattering, such as long-range Coulomb disorder scattering, is on average forward displaced in momentum space. A similar result is also attributed to a graphene structure. $^{33}$

Assuming that the change in the $\mathrm{SdH}$ amplitude with an applied electric field can be described in terms of electricfield induced electron heating, the temperature $T$ in Eq. (5) can be replaced by the electron temperature $T_{e}{ }^{2,3,5,26}$ Therefore, $T_{e}$ can be determined by comparing the relative amplitudes of the $\mathrm{SdH}$ oscillations measured as functions of the lattice temperature $\left(T=T_{L}\right)$ and the applied electric field $(F)$ using ${ }^{2,3,5}$

$$
\left[\frac{A\left(T_{L}, B_{n}\right)}{A\left(T_{L 0}, B_{n}\right)}\right]_{F=F_{0}}=\left[\frac{A\left(F, B_{n}\right)}{A\left(F_{0}, B_{n}\right)}\right]_{T_{L}=T_{L 0}} .
$$

Here $A\left(F, B_{n}\right)$ and $A\left(F_{0}, B_{n}\right)$ are the amplitudes of the oscillation peaks observed at a magnetic field $B_{n}$ and at electric fields $F$ and $F_{0}$, respectively. In order to obtain the electron temperature from the lattice temperature and electric-field dependencies of the amplitude of the $\mathrm{SdH}$ oscillations, the quantum lifetime has to be independent of both the lattice temperature and the applied electric field. Figure 7(b) shows the amplitudes of the $\mathrm{SdH}$ oscillations, normalized as described by Eq. (6), as functions of $F$ for the monolayer graphene sample. In Fig. 7, only the relative amplitudes at a given magnetic field $B_{n}$ are shown for clarity. A similar analysis that was conducted for all the $\mathrm{SdH}$ peaks that were

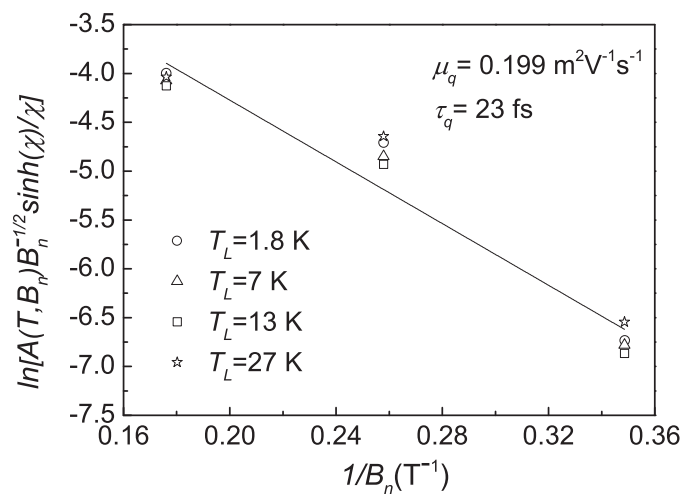

FIG. 8. Determination of the quantum lifetime in the graphene sample. The data points represented by symbols and the straight lines are the leastsquares fits of Eq. (6) to the experimental data, respectively.

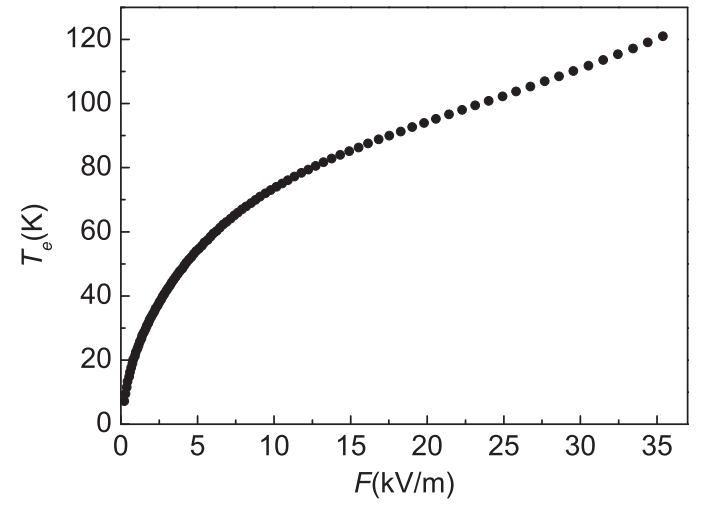

FIG. 9. Electron temperature $\left(T_{e}\right)$ versus the applied electric field $(F)$ for the graphene sample.

observed in the magnetic field range from 2 to $11 \mathrm{~T}$ has established that the relative amplitudes of $\mathrm{SdH}$ oscillations (and hence the electron temperatures) in our samples are essentially independent of a magnetic field. This indicates that the magnetic field used in our experiments does not significantly alter the energy relaxation processes of hot electrons.

Electron temperatures $\left(T_{e}\right)$ for the monolayer graphene sample as obtained by directly comparing the curves similar to those in Figs. 7(a) and 7(b) are plotted as a function of the applied electric field in Fig. 9. The SdH oscillations measured for the monolayer graphene sample decrease rapidly with the increase of the applied electric field and become vanishingly small for $F>35 \mathrm{kV} \mathrm{m}^{-1}$ [see Figs. 5(b) and 7(b)]. The electron temperature determined for this sample rises quickly with increasing $F$.

In the steady state, the power loss from hot electrons by the emission of acoustic phonons is equal to the power supplied by the applied electric field, which can be calculated using the energy balance equation ${ }^{2,3}$

$$
P=e \mu_{H} F^{2},
$$

where $P$ and $F$ are the energy loss (or energy supply) rate per electron and applied electric field, respectively. In order to obtain the electron temperature from the lattice temperature and electric-field dependencies of the amplitude of the SdH oscillations, both the quantum lifetime and the transport mobility have to be independent of both the lattice temperature and the applied electric field. It is found that $\mu_{H}$ is independent of both the lattice temperature and the applied electric field in the ranges of the measurements. The power loss versus electron temperature is plotted in Fig. 10. The electron temperature dependence of the power loss was found to be similar with the previous reports on energy relaxation by hot electrons in graphene samples. ${ }^{9,18}$

The variation of the power loss per electron with electron temperature in the acoustic phonon regime has often been approximated by the relationship

$$
P=A\left(T_{e}^{\gamma}-T_{L 0}^{\gamma}\right),
$$

where $A$ is the proportionality constant, which depends on the elastic moduli of the matrix, the coupling constants, and the 2D carrier density. Theoretical calculations of the 


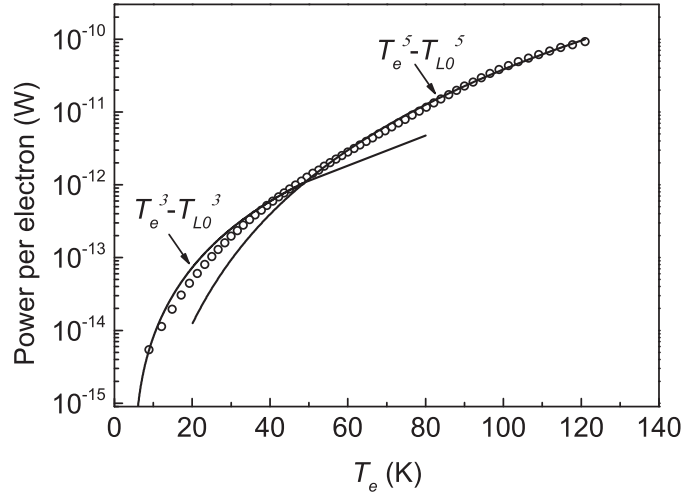

FIG. 10. Electron temperature dependence of power loss per electron determined from $\mathrm{SdH}$ measurements. The open circles correspond to the experimental data. The full curves correspond to the power loss calculated using Eq. (9).

acoustic phonon assisted energy loss rates of hot electrons in a $2 \mathrm{D}$ electron gas of single-subband occupancy predict $\gamma=1$ at high temperatures (when Maxwell-Boltzmann statistics is applicable and equipartition is assumed) and $\gamma=3$ (unscreened piezoelectric scattering), $\gamma=5$ (unscreened deformation potential and heavily-screened piezoelectric scatterings), and $\gamma=7$ (heavily screened deformation potential scattering) at low temperatures (see, for instance, Ref. 4). We found the exponent 3 in the range $1.8<T_{e}<50 \mathrm{~K}$ and 5 in the range $50<T_{e}<130 \mathrm{~K}$ by fitting Eq. (9) to the experimental data (Fig. 10). In all cases, a constant value for the exponent $\gamma$ is obtained over the temperature range (Fig. 10). This indicates that the experiments were carried out in the low-temperature regime and that the energy relaxation is due to acoustic phonon emission via mixed unscreened piezoelectric and deformation potential interactions.

The energy relaxation time $\left(\tau_{E}\right)$ for intrasubband processes can be obtained from the power loss measurements using $^{4}$

$$
P=\frac{\langle\hbar \omega\rangle}{\tau_{E}} \frac{\left(k_{B} T_{e}-k_{B} T_{L}\right)}{k_{B} T_{e}},
$$

where $\langle\hbar \omega\rangle=2^{1 / 2} \hbar V_{S} k_{F}$ is the acoustic-phonon energy averaged over the Fermi surface. Figure 10 shows the energy relaxation time as a function of the electron temperature

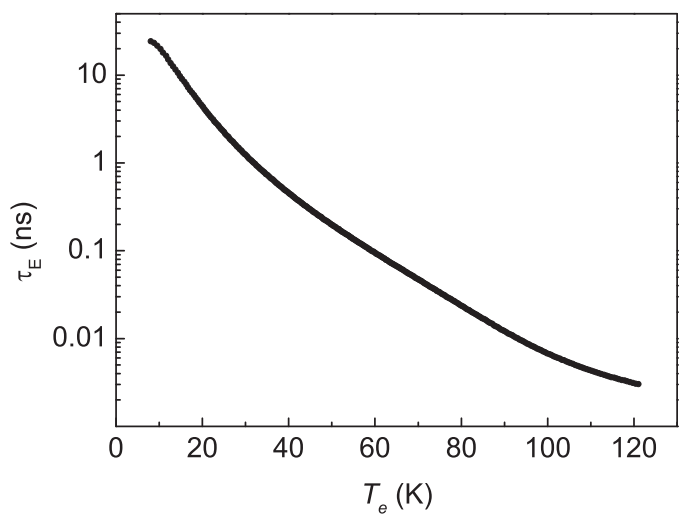

FIG. 11. Energy relaxation time $\left(\tau_{E}\right)$ versus $T_{e}$ for the graphene sample. for the graphene sample studied. The electron temperature dependence of the energy relaxation time was found to be similar with the previous reports on energy relaxation by hot electrons in graphene samples. ${ }^{9,18}$ However, as can be seen in Fig. 11, the energy relaxation due to acoustic phonons becomes faster at higher electron temperatures.

\section{CONCLUSIONS}

The carrier density $\left(N_{2 D}\right)$ and effective mass $\left(m^{*}\right)$ for electrons in the monolayer graphene have been determined from the Shubnikov de Haas ( $\mathrm{SdH}$ ) oscillations. The twodimensional (2D) carrier density and the $m^{*}$ have been obtained from the periods of the $\mathrm{SdH}$ oscillations and the temperature dependencies of the $\mathrm{SdH}$ amplitude, respectively.

The energy-loss rates, in the acoustic phonon regime, of $2 \mathrm{D}$ electrons in a monolayer graphene have also been investigated using $\mathrm{SdH}$ effect measurements. The experimental results were compared with the predictions of the current theoretical models for power loss in semiconductors. The energy relaxation of electrons is due to acoustic phonon emission via piezoelectric and deformation-potential scattering.

${ }^{1}$ S. D. Sarma, S. Adam, E. H. Hwang, and E. Rossi, Rev. Mod. Phys. 83, 407 (2011).

${ }^{2}$ H. Kahlert and G. Bauer, Phys. Status Solidi B 46, 535 (1971).

${ }^{3}$ G. Bauer and H. Kahlert, Phys. Rev. B 5, 566 (1972).

${ }^{4}$ B. K. Ridley, Rep. Prog. Phys. 54, 169 (1991).

${ }^{5}$ N. Balkan, H. Celik, A. J. Vickers, and M. Cankurtaran, Phys. Rev. B 52, 17210 (1995).

${ }^{6}$ K. S. Novoselov, A. K. Geim, S. V. Morozov, D. Jiang, M. I. Katsnelson, I. V. Grigorieva, S. V. Dubonos, and A. A. Firsov, Nature (London) 438, 197 (2005).

${ }^{7}$ Y. B. Zhang, Y. W. Tan, H. L. Stormer, and P. Kim, Nature (London) 438, 201 (2005).

${ }^{8}$ Z. Tan, C. Tan, L. Ma, G. T. Liu, L. Lu, and C. L. Yang, Phys. Rev. B 84, 115429 (2011).

${ }^{9}$ W.-K. Tse and S. D. Sarma, Phys. Rev. B 79, 235406 (2009).

${ }^{10}$ H. M. Dong, W. Xu, and R. B. Tan, Solid State Commun. 150, 1770 (2010).

${ }^{11}$ H. M. Dong, W. Xu, and F. M. Peeters, J. Appl. Phys. 110, 063704 (2011).

${ }^{12}$ K. S. Bhargavi and S. S. Kubakaddi, Physica E 44, 1766 (2012).

${ }^{13}$ D. Sun, Z.-K. Wu, C. Divin, X. Li, C. Berger, W. A. de Heer, P. N. First, and T. B. Norris, Phys. Rev. Lett. 101, 157402 (2008).

${ }^{14}$ J. M. Dawlaty, S. Shivaraman, M. Chandrashekhar, F. Rana, and M. G. Spencer, Appl. Phys. Lett. 92, 042116 (2008).

${ }^{15}$ B. A. Ruzicka, S. Wang, L. K. Werake, B. Weintrub, K. P. Loh, and H. Zhao, Phys. Rev. B 82, 195414 (2010).

${ }^{16}$ J. Voutilainen, A. Fay, P. Hkkinen, J. K. Viljas, T. T. Heikkil, and P. J. Hakonen, Phys. Rev. B 84, 045419 (2011).

${ }^{17}$ A. S. Price, S. M. Hornett, A. V. Shytov, E. Hendry, and D. W. Horsell, Phys. Rev. B 85, 161411 (2012).

${ }^{18}$ A. M. R. Baker, J. A. Alexander-Webber, T. Altebaeumer, and R. J. Nicholas, Phys. Rev. B 85, 115403 (2012).

${ }^{19}$ S. S. Kubakaddi, Phys. Rev. B 79, 075417 (2009).

${ }^{20}$ D. S. Lee, C. Riedl, B. Krauss, K. von Klitzing, U. Starke, and J. H. Smet, Nano Lett. 8, 4320 (2008).

${ }^{21}$ J. C. Burton, L. Sun, F. H. Long, Z. C. Feng, and I. T. Ferguson, Phys. Rev. B 59, 7282 (1999).

${ }^{22}$ A. C. Ferrari, J. C. Meyer, V. Scardaci, C. Casiraghi, M. Lazzeri, F. Mauri, S. Piscanec, D. Jiang, K. S. Novoselov, S. Roth, and A. K. Geim, Phys. Rev. Lett. 97, 187401 (2006).

${ }^{23}$ G. Weimann, Festkorperprobleme: Adv. Solid State Phys. 26, 231 (1986).

${ }^{24}$ S. Hiyamizu, J. Saito, K. Nanbu, and T. Ishikawa, Jpn. J. Appl. Phys. 22, L609 (1983).

${ }^{25}$ E. E. Mendez, P. J. Price, and M. Heiblum, Appl. Phys. Lett. 45, 294 (1984).

${ }^{26}$ E. Tiras, M. Cankurtaran, H. Celik, and N. Balkan, Phys. Rev. B 64, 085301 (2001). 
${ }^{27}$ V. P. Gusynin and S. G. Sharapov, Phys. Rev. Lett. 95, 146801 (2005).

${ }^{28}$ W. A. de Heer, C. Berger, X. Wu, M. Sprinkle, Y. Hu, M. Ruan, J. A. Stroscio, P. N. First, R. Haddon, B. Piot et al., J. Phys. D: Appl. Phys. 43, 374007 (2010).

${ }^{29}$ H. L. Störmer, R. Dingle, A. C. Gossard, W. Wiegmann, and M. D. Sturge, Solid State Commun. 29, 705 (1979).
${ }^{30}$ E. Tiras, M. Cankurtaran, H. Celik, A. Boland-Thoms, and N. Balkan, Superlattices Microstruct. 29, 147 (2001).

${ }^{31}$ E. Tiras, N. Balkan, S. Ardali, M. Gunes, F. Fontaine, and A. Arnoult, Philos. Mag. 91, 628 (2011).

${ }^{32}$ H. Celik, M. Cankurtaran, A. Bayrakli, E. Tiras, and N. Balkan, Semicond. Sci. Technol. 12, 389 (1997).

${ }^{33}$ X. Hong, K. Zou, and J. Zhu, Phys. Rev. B 80, 241415 (2009). 